Original Research

\title{
The Role of General Medicine in the Evaluation of Frailty in the Elderly Population: Definition of a Standardized Instrument for the Correct Framing of Frailty and Comparison with Currently Existing Instruments for Stratification of Clinical Risk
}

Fabio Robusto ${ }^{1,}{ }^{*}$, Giovanni Colucci ${ }^{1}$, Maria Zamparella ${ }^{1}$, Enrico Maria Pellegrini ${ }^{1}$, Brigida Martucci 1, Pasquale lacovazzo 1, Enza Colucci 1, Martino Minardi 1, Cristina Giliberti 2, Vito Bossone ${ }^{3}$, Vito Lepore ${ }^{4}$, Stefano Ivis ${ }^{3}$

1. MedOnLine-Statte, Statte (TA), Italy; E-Mails: fabiorobusto@libero.it; drgcolucci@libero.it; zamparella.maria@gmail.com; enricopellegrini.m@gmail.com; brigidamartucci95@gmail.com; iacovazzo.pasquale@libero.it; enza.colucci@libero.it; minarm@libero.it

2. Senior Lecturer in Italian Rice University, Houston, Texas, USA; E-Mail: cristinagiliberti@hotmail.com

3. Keiron AsMeG, Verona, Italy; E-Mails: vitobossone@tiscali.it; stefanoivis@gmail.com

4. Neurologist, former researcher, department of Neuroscience, Univerisity of Bari, Bari, Italy; EMail: leporevito792@gmail.com

* Correspondence: Fabio Robusto; E-Mail: fabiorobusto@libero.it

Academic Editor: Calogero Caruso

Special Issue: Depression in the Elderly

OBM Geriatrics

2022, volume 6 , issue 1

doi:10.21926/obm.geriatr.2201192
Received: September 08, 2021

Accepted: February 22, 2022

Published: March 04, 2022

\section{Abstract}

Frailty is a chronic condition that increases the vulnerability to stressogenic factors and prevents the patient from returning to the preceding condition of homeostasis. This increases the risk of negative outcomes and progressively brings the patient toward disability, leading to higher use of healthcare resources. Clinical risk stratification systems can generally be useful for identifying frail patients from the standpoint of a healthcare 
system, though General Practitioners (GPs) assume a key and irreplaceable role in the definition and correct diagnosis of frailty. This study developed a standardized instrument (called SVaFra) for the definition of frailty in the elderly population in a general medicine setting and compared it with a few clinical risk stratification tools that have already been validated and are in wide use. In addition, the impact of the application of SVaFra on healthcare outcomes was evaluated. A scientific board composed of experienced GPs, biomedical engineers, and other healthcare professionals, involved in the management of patients suffering from frailty, developed a framework in the form of a questionnaire for the evaluation of frailty by creating four principal groupings of the components that characterize it (clinical complexity, disability, family environment, and management complexity). An observational study, involving 98 GPs from four Italian regions who filled out the questionnaire, was then developed. The doctors were asked to provide a judgment for the four frailty components and the overall frailty. Additionally, a cohort of patients was stratified by applying Charlson Comorbidity Index (CCI) and Drug Derived Complexity Index (DDCI) to administrative databases. The utilization of healthcare resources in the year following the administration of the SVaFra framework with this population was compared with a control group with similar clinical or demographic characteristics. A total of 1,305 frail geriatric patients were identified (males $36.0 \%$; mean age $83.1 \pm 8.52$ years). Regarding the four principal areas used by the GPs to formulate a frailty judgment, the clinical categorization "moderate-severe" was most frequently noted (57.0\%). The GPs then specified the following most frequent pathologies: arterial hypertension (76.4\%), congestive heart failure (31.5\%), dementia (30.7\%), diabetes (29.9\%), cardiac arrhythmia (27.6\%), major depression $(25.2 \%)$, stroke $(22.0 \%)$, respiratory insufficiency $(22.0 \%)$, chronic renal insufficiency (12.6\%), management complexity (48.3\%), disability $(43.2 \%)$, and family environment (23.8\%). For 165 subjects (12.6\%), the GPs expressed an overall frailty judgment of "severe" based on management complexity and disability. Record linkage with administrative databases was possible in 102 cases. The presence of a $\mathrm{CCl}$ score of $>0$ was recorded in only $20(15.3 \%)$ patients identified as frail by GPs, while high DDCl scores were recorded for $88(86.3 \%)$ patients. As for the utilization of healthcare resources, a net reduction of healthcare costs, especially those associated with emergency services, was observed for the population characterized as frail by GPs as compared with the control group with similar clinical or demographic characteristics. The SVaFra instrument was simple to apply, with transferability for the individualization and characterization of frail patients in diverse healthcare realities. However, $\mathrm{CCl}$, which was useful for the stratification of clinical risk profiles, classified the majority of patients who were identified as frail by GPs as low risk. On the other hand, high scores were identified by DDCl for the majority of frail patients. The simple focus of GPs on the problem of frailty obtained by the administration of the SVaFra framework led to a reduction of healthcare costs due to emergency room treatments. Thus, the development of adequate diagnostic and therapeutic pathways to be developed about healthcare systems based on the use of SVaFra may result in more careful and accurate management of frail patients in the future.

\section{Keywords}

Frailty; drug use; administrative databases; risk stratification; general practice 


\section{Introduction}

Social and demographic changes and the promotion of preventive and curative strategies for chronic pathologies have resulted in increased frailty among the elderly [1], who are characterized by health problems that are not only clinical but also social and charitable [2]. Frailty is defined as a condition of vulnerability to acute stressogenic events capable of provoking the loss of selfsufficiency. The close relationship between frailty and the healthcare indicators (from inappropriate use of health services to polypharmacotherapy) has led to the development of recent directives of the European Union (EU) for the sustainability of national healthcare systems [3].

Previous studies have used a variety of statistical tools to identify patients at high risk. In these tools, clinical and/or socio-economic data are integrated into predictive models [4-15]. The development of tools for risk stratification by integrating diverse sources of administrative data has allowed for the grouping of the assisted population in levels of healthcare complexity, such as the risk of mortality, repeated hospitalizations, or other uses of healthcare resources [16]. Such predictive tools as well as the Chronic Care Model (CCM) and Diagnostic-Therapeutic Care Pathways (DTCPs), cannot ignore the involvement of General Medicine. The doctors of General Medicine, responsible for the regional management of assisted patients, are indispensable for the planning and defining the management of chronic conditions, not only as producers of data but also as part of the development, validation, and application of indexes capable of stratifying the risk profile of the population.

The introduction of the concept of frailty to General Medicine and the case-mix of patient populations is indispensable in regional performance standards, appropriateness of treatment, and recourse to emergency services. It also emerged in the context of territorial healthcare services - the problem of how to describe and stratify more precisely this frequent and undefined "clinical-socio-functional" condition, recognized at the time only by the specialist scientific community who limited themselves to the usual clinical aspects. It was clear that only a simple yet precise description of the multi-pronged problem would permit the transferability of data and opening up of clinical, government, and administrative research-conditions considered indispensable for the improvement of social-medical care [17].

The SVaFra framework is put along this path, providing the contribution of General Medicine to healthcare needs that characterize the frail population and initial analysis on the appropriateness of the modalities of the healthcare system. A scientific board composed of general practitioners, biomedical engineers, and other healthcare professionals have developed and validated a tool to standardize the definition of frailty by characterizing its different areas of vulnerability in an evaluation form.

\subsection{Aims}

The main objective of this study is the development and validation of a new stratification tool to identify and stratify frail patients. Moreover, the effect of the implementation of this 
stratification tool on healthcare direct costs was evaluated in a sub-cohort of patients through a matched pair case-control study.

\section{Materials and Methods}

\subsection{The SVaFra Framework}

The growing importance of management aspects, such as the management of domestic concerns and primary assistance, and workplace sustainability in the care of elderly patients afflicted by frailty, has brought about the creation of a working group of General Practitioners (GPs) and management engineers. With the logic of research-action, a series of focus groups among experts have shared that the complexity of the frail patient's condition required a summary description of various criteria, both subjective and objective. The first subjective criterion was represented by the judgment of frailty (clinical and predictive stability) and integrated patient needs. The responsibility for both evaluations was entrusted to the primary care physician based on the assumption that he or she best knows the history of the frailty of the patient and the consequences.

The other criterion for assessing patient needs was objective, and similar to the TNM Classification for neoplasms, it was distributed amongst the variables clinical, disability, family context, and management complexity. Here too, each variable was stratified as slight, medium, and severe (numerically represented from one to three). Based on this checklist, an evaluation sheet of the frail elderly called "SVaFra" was developed. Once the conceptualization of the problem and the tool to represent were finalized, dissemination of the contents to the scientific community of the GPs (General Practitioners) was performed. During the course, the assumptions, contents, and expected outcomes of using the framework or card were illustrated; some suggestions for improvement were accepted. In a logic of co-construction, the definition of frailty by family doctors, which was missing until then, was adopted. A tool was thus developed that can define frailty and its dimensions in complex elderly patients through the contribution of General Medicine. The definition and reading of the care needs perceived by the GPs have been expressed through the formulation of a module. Through a numerical scale, this module could quantify the state of frailty perceived by the GPs and define the determinants that led to express their judgment.

\subsection{Population and Risk Stratification}

The SVaFra card was administered to patients aged $\geq 65$ years who were in charge of 98 GPs in four Italian regions (Veneto, Puglia, Lazio, and Sicily); the patients had at least two of the following conditions:

- chronically affected by multiple pathologies,

- unstable state of health,

- state of relapsing psycho-physical disability,

- $\quad$ socio-economic problems (loneliness, low income, and relational difficulties),

- $\quad$ aged $\geq 65$ years who, in the doctor's reasoned opinion, deserves to be included, and

- all patients aged $\geq 65$ years IN TEMPORARY ADI (Temporary Integrated Home Care). 
First, the GPs were asked to express an independent clinical judgment on the degree of overall "frailty" perceived; the following degrees were used:

- 0 (absence of frailty), requiring implementation of prevention activities,

- 1 (mild frailty), a reversible condition that requires care and rehabilitation,

- 2 (moderate fragility), an irreversible condition that requires personalized management, and

- 3 (severe frailty), an irreversible and terminal condition that requires palliative care and accompaniment.

After that, the frailty of the patient was classified according to four aspects, each with the following four degrees of judgment:

1. Health area:

- S0: patients without chronic diseases,

- S1: patients with one or more asymptomatic or symptomatic pathologies adequately treated,

- S2: patients with one or more pathologies not controlled by therapy or with one or more pathologies at the maximum of their severity, and

- Unstable S3: clinical problems that require medical monitoring, instrumental nursing, non-programmable, and occur one or more times per week, but not daily.

2. Physical or cognitive-behavioral disability:

- D0: absence of cognitive-behavioral problems,

- D1: needs support (e.g mild mental deterioration, significantly impaired work and social activities, the capacity for autonomous life remains, with adequate personal hygiene and relatively preserved critical capacity),

- D2: needs structured support (wheelchair), moderate mental deterioration, independent living becomes risky, and a certain degree of supervision is necessary, and

- D3: needs care (bed), severe mental deterioration, the activities of daily life are so compromised that continuous supervision is necessary, e.g., the patient is unable to maintain a minimum of personal hygiene or largely incoherent or mute.

3. Management complexity:

- G0: absence of management problems,

- G1: low management complexity, patient in ADP, clinically stable, collaborating (compliant) and with sufficient autonomy, need periodic checking up by an operator,

- G2: medium management complexity, patient in ADI, with problematic clinical stability, or not reliable in collaboration (non-compliant), or with insufficient autonomy for the treatment of his/her clinical condition, needs a periodic checkup by more than one operator, and

- G3: high management complexity, patient in complex ADI, simultaneously presents two of the factors of management complexity (problematic clinical stability, unreliable 
collaboration [non-compliant], and dependence on care), requires integrated and planned to check up by several operators.

\section{Environmental context:}

- C0: good environmental support,

- C1: the spouse and/or relatives are available but can provide assistance limited to the essential (cohabitants with various personal difficulties or not cohabiting with conditioning distances or availability), any private assistance would compromise the economic capacity and the dwelling, without external or internal architectural barriers,

- C2: the spouse and/or relatives have difficulty in assisting, insufficient economic availability for any private assistance, and housing not entirely suited to the situation, and

- C3: has no relatives or relatives are not able to assist, housing with external or internal architectural barriers.

Since the GPs were asked to express an overall judgment of frailty, the combination of the scores of the four dimensions described above (of which a graphic representation is available in Figure 1) does not influence the score but are independent factors in qualifying it. Therefore, among health, disability, management complexity, and environmental areas, the factors most influencing the GP's clinical judgment in the definition of frailty will be analyzed.

SCHEDA DI VALUTAZIONE DELL'ANZIANO FRAGILE (SVAFRA)

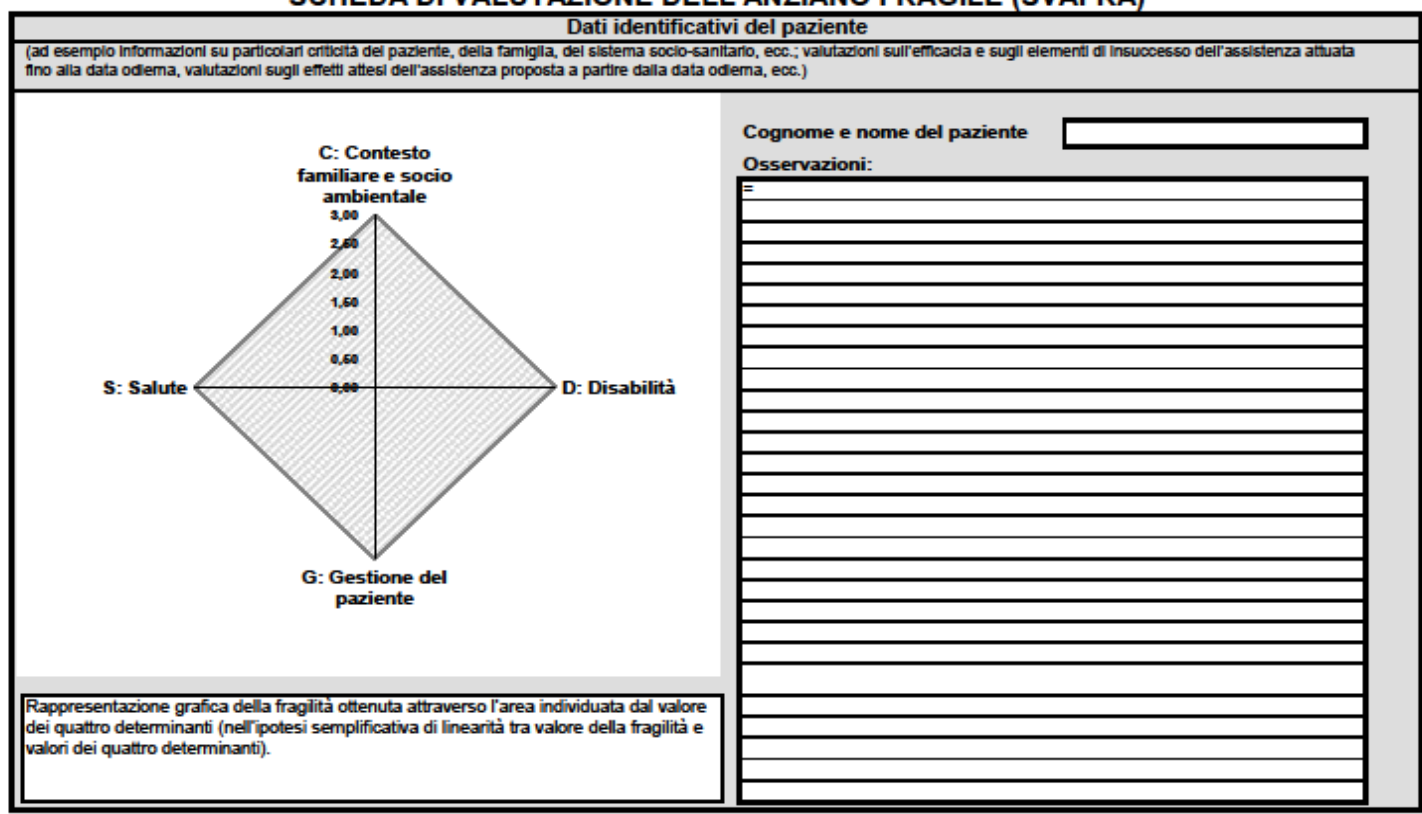

Figure $1 \mathrm{SVaFra}$ card: graphical presentation of frailty as an area underlying the use of the dimensions considered in its definition.

In addition, in a cohort of subjects for whom it was possible to perform record linkage procedures based on administrative databases, the cost produced by the use of healthcare resources and survival and access to total hospital and emergency admissions in the period 
following the compilation of the SVaFra form were evaluated. To address this purpose, a control group was selected, with the same clinical or demographic characteristics but not indicated by the GPs as frail, through a matched-pair analysis using the following covariates:

- Charlson Comorbidity Index (CCI), calculated by applying the ICD9-CM coding to the diagnoses of pathologies present in the scenes of hospital discharge (SDO) in the 12 months before the compilation of the SVaFra form,

- $\quad$ the Drug Derived Complexity Index (DDCI), applied to the pharmaceutical prescriptions produced in the 12 months before the compilation of the SVaFra form,

- gender, and

- age.

\subsection{GPS Statistical Analyses}

Scores from the application of the SVaFra card were reported as a mean and standard deviation; any correlations between the scores of the four aspects and the overall judgment of frailty were evaluated through linear regression. The coefficient of determination was evaluated to determine the proportion between the variability of the data and the correctness of the statistical model used.

The use of healthcare resources was evaluated during the 12 months following the completion of the SVaFra form or until the previous date of cancellation from the regional assisted registry for transfer or death. Overall survival, healthcare expenditures, and recourse to planned and unplanned hospitalizations were evaluated through time-to-event analysis. Results were considered statistically significant when $p<0.05$. All statistical analyses were performed using the SAS Software Release 9.4 (SAS Institute, Cary, NC).

\section{Results}

A total of 1,305 "frail" patients ( $M$ 36.7\%) with an average age of $83.1 \pm 8.2$ years and treated during 2016 were included. The $98 \mathrm{GPs}$, who enrolled a minimum of five to a maximum of 43 patients, expressed for each of them a judgment of severity concerning the problems related to the different dimensions that characterize frailty (Table 1).

Table 1 Classification according to the SVaFra card for each of the four dimensions of frailty.

\begin{tabular}{lll}
\hline Health & $\mathrm{N}$ & $\%$ \\
\hline S0 & 1 & 0.1 \\
S1 & 560 & 42.9 \\
S2 & 565 & 43.3 \\
S3 & 179 & 13.7 \\
Disability & $\mathrm{N}$ & $\%$ \\
\hline
\end{tabular}




\begin{tabular}{lll}
\hline D0 & 147 & $11.3 \%$ \\
D1 & 595 & $45.6 \%$ \\
D2 & 339 & $26.0 \%$ \\
D3 & 224 & $17.2 \%$ \\
Management complexity & $\mathrm{N}$ & $\%$ \\
G0 & 172 & 13.2 \\
G1 & 502 & 38.5 \\
G2 & 495 & 37.9 \\
G3 & 136 & 10.4 \\
Environmental context & $\mathrm{N}$ & $\%$ \\
C0 & 484 & $37.1 \%$ \\
C1 & 510 & $39.1 \%$ \\
C2 & 235 & $18.0 \%$ \\
C3 & 76 & $5.8 \%$ \\
\hline
\end{tabular}

The most frequent element of frailty is linked to problems of disability and health and management complexity, while the environmental context is a less frequent variable. The overall assessment of frailty at baseline is shown in Table 2.

Table 2 Overall assessment of frailty expressed by the GPs.

\begin{tabular}{lll}
\hline Frailty & Frequency & Percent \\
\hline F0 & 24 & $1.8 \%$ \\
F1 & 265 & $20.3 \%$ \\
F2 & 851 & $65.2 \%$ \\
F3 & 165 & $12.6 \%$ \\
\hline
\end{tabular}

Even if the discretionary judgment of the overall degree of frailty was formulated independently from the scores of the four considered dimensions, a progressive increase in the average size scores is noted for each degree of frailty. This gradient is more marked for the dimensions of health, disability, and management complexity. A similar figure is reflected in the ratio between the scores of frailty and those of the four dimensions in linear regression. Moreover, a statistical significance and a value of the coefficient of determination greater in the relationship of direct proportionality between frailty and dimensions of health and disability were observed (Table 3). 
Table 3 Average value, coefficient of determination, and statistical significance of the proportionality relationship between the four dimensions and the overall frailty judgment perceived by the GPs.

\begin{tabular}{|c|c|c|c|c|}
\hline Frailty & Health & Disability & Management & Context \\
\hline FO & 1.19 (SD 0.54) & 0 (SD 0) & 0.25 (SD 0.58) & 0 (SD 0) \\
\hline $\mathrm{F} 1$ & 1.15 (SD 0.37) & 0.66 (SD 0.54) & 0.84 (SD 0.84) & 0.65 (SD 0.70) \\
\hline F2 & 1.69 (SD 0.64) & 1.54 (SD 0.80) & 1.44 (SD 0.73) & 1.44 (SD 1.73) \\
\hline F3 & 2.49 (SD 0.61) & 2.29 (SD 0.81) & 2.06 (SD 0.78) & 0.99 (SD 0.96) \\
\hline \multicolumn{2}{|c|}{ Dimension } & \multicolumn{2}{|c|}{ Coefficient of determination } & $\mathrm{p}$ \\
\hline \multicolumn{2}{|l|}{ Health } & \multicolumn{2}{|c|}{$0.27( \pm 0.03)$} & $<0.001$ \\
\hline \multicolumn{2}{|c|}{ Disability } & \multicolumn{2}{|c|}{$0.25( \pm 0.02)$} & $<0.001$ \\
\hline \multicolumn{2}{|c|}{ Management complexity } & \multicolumn{2}{|c|}{$0.10( \pm 0.02)$} & $<0.001$ \\
\hline \multicolumn{2}{|c|}{ Environmental context } & \multicolumn{2}{|c|}{$0.05( \pm 0.04)$} & 0.01 \\
\hline
\end{tabular}

The availability of historical series of administrative databases of one of the regions included in the study, with adequate territorial and temporal coverage, allowed record linkages between the clinical data of the GPs and the administrative ones. This led to the identification of a cohort of 120 frail patients (with the following pathologies: arterial hypertension: $76.4 \%$, heart failure: 31.5\%, dementia: $30.7 \%$, diabetes mellitus: $29.9 \%$, cardiac arrhythmias: $27.6 \%$, major depression: $25.2 \%$, stroke: $22.0 \%$, respiratory failure: $22.0 \%$, and chronic renal failure: $12.6 \%$ ) for whom administrative information was available. The Hospital Discharge Records (SDOs) produced in the 12 months before the compilation of the SVaFra form by the patients were selected, and the $\mathrm{CCl}$ (based on the ICD9 encoding of the diagnoses) was calculated. Similarly, territorial pharmaceutical prescriptions, produced in the 12 months preceding the index date, were selected, and the DDCI score was calculated through the ATC coding of the prescribed drugs. The presence of a $\mathrm{CCl}>0$ score was recorded in only $16(13.3 \%)$ patients defined as frail by the GPs, while higher DDCI scores were found in $91(75.8 \%)$ patients.

In 2016 among the 40-year-old population, a 1-to-1 matched-pair analysis was performed, selecting a non-oncological patient with the same age, gender, $\mathrm{CCl}$, and the $\mathrm{DDCl}$ scores of the SVaFra cohort. The characteristics of the population before and after the selection performed in the matched-pair-analysis is provided in Table 4. 
Table 4 Comparison of clinical-demographic characteristics among the general population, study cohort, and control cohort in the matched-pair analysis.

\begin{tabular}{|c|c|c|c|}
\hline Characteristics & $\begin{array}{l}\text { Inhabitants aged } 40 \\
\text { or more }\end{array}$ & Study cohort & Control cohort \\
\hline Total, $n$ & $2 \cdot 236^{\circ} 052$ & 120 & 120 \\
\hline Gender, $n(\%)$ & $1^{\circ} 041^{\circ} 731(46.6)$ & $34(14.2)$ & $34(14.2)$ \\
\hline \multicolumn{4}{|l|}{ Age } \\
\hline Mean (STD) & $60.3( \pm 13.9)$ & $81.2( \pm 7.3)$ & $80.3( \pm 13.9)$ \\
\hline Range & $40.0-110.5$ & $64.4-98.9$ & $63.1-102.5$ \\
\hline \multicolumn{4}{|c|}{ Charlson Index, n (\%) } \\
\hline 0 & $2 \cdot 092784(93.6)$ & $104(86.7)$ & 104 (86.7) \\
\hline $1-2$ & $78 \cdot 966(3.5)$ & $11(9.2)$ & $11(9.2)$ \\
\hline $3-4$ & $38 \cdot 952(1.7)$ & $4(3.3)$ & $4(3.3)$ \\
\hline$\geq 5$ & $25 \cdot 350(1.1)$ & $1(0.8)$ & $1(0.8)$ \\
\hline \multicolumn{4}{|l|}{ DDCl, n (\%) } \\
\hline$\leq 1$ & $17000^{\circ} 486(76.1)$ & $29(24.2)$ & $29(24.2)$ \\
\hline $2-3$ & $322 \cdot 196(14.4)$ & $38(31.7)$ & $38(31.7)$ \\
\hline $4-5$ & $122^{\circ} 051(5.5)$ & $24(20.0)$ & $24(20.0)$ \\
\hline $6-7$ & $56.865(2.5)$ & $13(10.8)$ & $13(10.8)$ \\
\hline 8-9 & $223318(1.0)$ & $12(10.0)$ & $12(10.0)$ \\
\hline$\geq 10$ & $12 \cdot 136(0.5)$ & $4(3.3)$ & $4(3.3)$ \\
\hline
\end{tabular}

The analysis of the health resources practices during 2016 showed a sharp decrease in hospital spending in the population identified as frail by GPs when compared to the control population with the same clinical-demographic characteristics. A reduction in hospital spending in SVaFra patients of the same mortality rate can be observed from the data reported in Table 5 . In this cohort, an average hospital spending per person per year of 658.5 euros was observed against the 812.4 euros of the control court (IRR $0.84 ; 95 \% \mathrm{Cl} 0.83-0.84$ ). On the contrary, no difference was observed in the pharmaceutical spending between cases (113.5 euros per person per year) and checkups (115.9 euros per person per year). Moreover, a significant decrease was observed in the number of emergency hospitalizations in the cohort of cases $(0.06$ urgent hospitalizations per person per year) compared to the control cohort ( 0.12 urgent hospitalizations per person per year), presenting an incidence ratio rate (IRR) of $0.50(95 \% \mathrm{Cl}: 0.22-0.79)$. On the contrary, a nonsignificant increase was observed in planned hospitalizations per person per year ( 0.11 versus $0.08)$, with an IRR of 1.38 (95\% Cl: 0.64-2.98).

Table 5 Events and costs (in Euro) per person per year in 2016. Comparison between SVaFra population and the control group.

$\begin{array}{llll}\text { Deaths Hospital costs } & \begin{array}{l}\text { Pharmaceutical } \\ \text { costs }\end{array} & \begin{array}{l}\text { Unplanned } \\ \text { hospitalizations }\end{array} & \text { A\&E access }\end{array}$

$\begin{array}{llllllll}I R & I R R(C I) & I R & I R R(C I) & I R & I R R(C I) & I R & I R R(C I)\end{array}$




\begin{tabular}{llllllllll}
\hline SVaFra & 13 & 658.5 & 0.84 & 113.5 & 1.00 & 0.11 & 1.38 & 0.06 & 0.50 \\
population & $(10.8 \%)$ & & $(0.83-0.84)$ & & $(0.99-1.01)$ & & $(0.64-2.98)$ & & $\begin{array}{l}(0.22- \\
0.79)\end{array}$ \\
& & & & & & & & & \\
Controls & 13 & 812.4 & 1 & 115.9 & 1 & 0.08 & 1 & 0.12 & 1 \\
& $(10.8 \%)$ & & & & & & & & \\
\hline
\end{tabular}

$\mathrm{IR}=$ Incidence Rate

IRR = Incidence Rate Ratio

\section{Discussion}

The sustainability of health systems in addressing aging and chronic care requires a rationalization of the delivery of health services about the emerging needs of the population. Therefore, several health systems have called for and promoted the development of statistical tools capable of predicting the risk of death and the inappropriate use of health systems. In a review, these tools were classified into the following three groups about the methodological approaches used [18]:

- "threshold models" [19, 20], such as the international prognostic index [21] or the CHADS2Vasc score [22], that examine specific variables in risk profile stratification and appear more accurate when used in specific clinical settings than their application in entire populations $[23,24]$,

- "clinical knowledge", in which doctors may be able to identify patients with conditions of greater frailty but are ineffective in predicting the risk of developing such conditions and present problems in standardization between the judgments of different operators [4], and

- "predictive models" that use regression models in the identification of variables and appear to be superior to previous models [25-27].

The data sources used in the development of these tools are represented by clinical archives, ad-hoc questionnaires administered to health professionals, or by data archives established or adapted to their use in the constitution of the statistical model.

The administrative databases, routinely produced by health systems to define the motivations, type, and costs of the service provided, contain "useful" information on the contact between an individual and a service of the social-health system, thereby allowing to recognize in that "service", not the simple use of it but a person with a need. The possibility of having comparable or integrable data of an entire district area would therefore make it possible to characterize the health balance of the entire population. Previous studies have reported a variety of statistical tools that are used on administrative databases to identify high-risk patients. In these tools, clinical and/or socio-economic data are integrated into predictive models [12-15]. Unfortunately, the administrative databases exhibit limits of integration and availability and also have important gaps related to clinical, social, anthropometric, and voluptuary information of which only the GPs are the depositories.

The SVaFra card is part of a path developed in the context of General Medicine to make frailty and the associated management difficulties measurable or qualifiable. The identification of the qualifying dimensions of frailty has allowed the evaluation of the most influential parameters 
affecting the judgment of the GPs - an essential data leading to the standardization of the judging criteria. The use of administrative databases for the stratification of the clinical risk profile has allowed the development of risk stratification tools on outcome variables, such as mortality and consumption of health resources, which can be considered as proxies of severity or burdensomeness $[10,13,16]$. The clinical data produced by General Medicine instead allow integration of the clinical complexity with sociodemographic variables that are difficult to acquire from different data sources; this approach also allows to evaluate frailty more completely. At the same time, this joint availability of clinical and administrative data has made it possible to evaluate the effects of the selection induced by the application of the SVaFra card on the use of health resources. Comparing the cohort understudy with a control population with the same clinical-demographic characteristics, a significant decrease in the average expenditure on hospitalizations and the average number of urgent hospitalizations per person per year was highlighted. This effect can be justified by the greater attention paid by the GPs in identifying the frail population induced by the application of the SVaFra card and the consequent prevention of acute complications by the adoption of appropriate clinical-care pathways.

\section{Conclusions}

Currently, no guidelines indicating an unequivocal path for the treatment and healthcare of frail patients exist. The card can be a tool in not only defining the frailty profile of the assisted population but also in evaluating the adequacy of the methods of assistance and promoting the development of integrated clinical care pathways for assistance to the frail population.

\section{Author Contributions}

Conceptualization, Fabio Robusto, Vito Lepore, Stefano Ivis, and Vito Bossone. Methodology, Fabio Robusto, Vito Lepore, Giovanni Colucci, and Pasquale lacovazzo. Software, Fabio Robusto and Enza Colucci. Validation, Maria Zamparella and Martino Minardi. Formal Analysis, Fabio Robusto. Investigation, Giovanni Colucci, Stefano Ivis, Cristina Giliberti, and Enrico Maria Pellegrini. Data curation, Fabio Robusto, Brigida Martucci, and Giovanni Colucci. Writing-original draft preparation, Fabio Robusto, Vito Lepore, Giovanni Colucci. All authors have read and agreed to the published version of the manuscript.

\section{Competing Interests}

The authors have declared that no competing interests exist.

\section{References}

1. Clegg A, Young J, lliffe S, Rikkert MO, Rockwood K. Frailty in elderly people. Lancet. 2013; 381: 752-762.

2. Commission of the European Communities. White paper-together for health: A strategic approach for the EU 2008-2013 [Internet]. Brussels: Commission of the European Communities; 2007. Available from: https://op.europa.eu/en/publication-detail/Lpublication/34f74e87-1c26-428f-ade3-b0225e1b7061. 
3. de Manuel Keenoy E, David M, Mora J, Prieto L, Domingo C, Orueta J, et al. Activation of stratification strategies and results of the interventions on frail patients of healthcare services (ASSEHS) dg sanco project no. 201312 04. Eur Geriatr Med. 2014; 5: 342-346.

4. Dudley RA, Medlin CA, Hammann LB, Cisternas MG, Brand R, Rennie DJ, et al. The best of both worlds? Potential of hybrid prospective/concurrent risk adjustment. Med Care. 2003; 41: 5669.

5. HARP Chronic Disease Management. [cited date 2006 April 21]. Available from: http://www.health.vic.gov.au/harp-cdm/harppubs.htm.

6. InterRAI. The integrated suite of instruments [Internet]. InterRAI. [cited date 2006 April 24]. Available from: http://www.interrai.org/section/view/?fnode=38.

7. High-impact User Manager (HUM) Tool [Internet]. [cited date 2006 April 06]. Available from: http://www.drfosterintelligence.co.uk/managementinformation/HUM/.

8. Patients at Risk of Rehospitalisation (PARR) case finding tool. [cited date 2006 April 05]. Available from: http://www.kingsfund.org.uk/health topics/patients at risk/index.html.

9. King's Fund. [cited date 2005 September 03]. Available from: http://www.kingsfund.org.uk/health topics/predictive risk.html.

10. Wennberg JE, Fisher ES. Finding high quality, efficient providers for value purchasing cohort methods better than methods based on events. Med Care. 2002; 40: 853-855.

11. Boult C, Dowd B, McCaffrey D, Boult L, Hernandez R, Krulewitch H. Screening elders for risk of hospital admission. J Am Geriatr Soc. 1993; 41: 811-817.

12. Pacala JT, Boult C, Boult L. Predictive validity of a questionnaire that identifies older persons at risk for hospital admission. J Am Geriatr Soc. 1995; 43: 374-377.

13. Falasca P, Berardo A, Tommaso FD. Development and validation of predictive MoSaiCo (Modello Statistico Combinato) on emergency admissions: Can it also identify patients at high risk of frailty? Ann Ist Super Sanita. 2011; 47: 220-228.

14. Adie E, Baird D, Bridger P, Buchanan S, Guthrie C, Hattie J, et al. SPARRA: Scottish patients at risk of readmission and admission 2006. Available from: www.isdscotland.org/healthtopics/health-and-social-community-care/SPARRA/SPARRAhistory/SPARRA report.pdf.

15. Wennberg MD, Siegel M, Darin B, Filipova N, Russel R, Kenney L, et al. Combined predictive model. Final report \& technical documentation [Internet]. London: King's Fund; 2006. Available from: $w$ ww.kingsfund.org.uk/document.rm?id=6745.

16. Robusto F, Lepore V, D'Ettorre A, Lucisano G, De Berardis G, Bisceglia L, et al. The drug derived complexity index (DDCl) predicts mortality, unplanned hospitalization and hospital readmissions at the population level. PLoS One. 2016; 11: e0149203.

17. Sirven N, Rapp T. The cost of frailty in France. Eur J Health Econ. 2017; 18: 243-253.

18. Curry N, Billings J, Darin B, Dixon J, Williams M, Wennberg D. Predictive risk project. Literature review [Internet]. London: The King's Fund; 2005. Available from:

https://www.kingsfund.org.uk/sites/default/files/field/field document/predictive-riskliterature-review-june2005.pdf.

19. Cousins MS, Liu Y. Cost savings for a preferred provider organization population with multicondition disease management: Evaluating program impact using predictive modeling with a control group. Dis Manag. 2003; 6: 207-217.

20. Castlefields Health Centre: Chronic Disease management. [cited date 2005 August 02]. Available from: http://www.natpact.nhs.uk/uploads/Castlefields\%20Report.doc. 
21. Cousins MS, Shickle LM, Bander JA. An introduction to predictive modeling for disease management risk stratification. Dis Manag. 2002; 5: 157-167.

22. Coppens M, Eikelboom JW, Hart RG, Yusuf S, Lip GY, Dorian P, et al. The $\mathrm{CHA}_{2} \mathrm{DS} \mathrm{S}_{2}-\mathrm{VASc}$ score identifies those patients with atrial fibrillation and a $\mathrm{CHADS}_{2}$ score of 1 who are unlikely to benefit from oral anticoagulant therapy. Eur Heart J. 2013; 34: 170-176.

23. National Public Health Service for Wales. Rapid overview of the evidence on the use of predictive risk tools in chronic disease management [Internet]. Cardiff: National Public Health Service for Wales; 2006. Available from:

https://www2.nphs.wales.nhs.uk/healthserviceqdtdocs.nsf.

24. Matrix Research and Consultancy. Learning distillation of chronic disease management programmes in the UK. London: NHS Modernisation Agency; 2004.

25. Zekry D, Valle BH, Graf C, Michel JP, Gold G, Krause KH, et al. Prospective comparison of 6 comorbidity indices as predictors of 1-year post-hospital discharge institutionalization, readmission, and mortality in elderly individuals. J Am Med Dir Assoc. 2012; 13: 272-278.

26. Kojima G, Iliffe $S$, Walters K. Frailty index as a predictor of mortality: A systematic review and meta-analysis. Age Ageing. 2018; 47: 193-200.

27. Shi SM, Olivieri-Mui B, McCarthy EP, Kim DH. Changes in a frailty index and association with mortality. J Am Geriatr Soc. 2021; 69: 1057-1062.

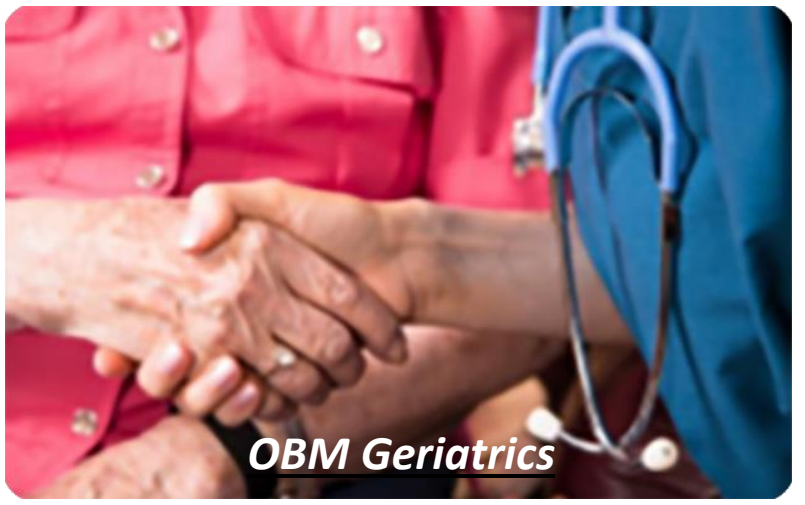

Enjoy OBM Geriatrics by:

1. Submitting a manuscript

2. Joining in volunteer reviewer bank

3. Joining Editorial Board

4. Guest editing a special issue

For more details, please visit: http://www.lidsen.com/journals/geriatrics 